\title{
Dynamic backcalculation with different load-time histories
}

\author{
Madsen, Stine Skov; Levenberg, Eyal
}

\section{Published in:}

Road Materials and Pavement Design

Link to article, DOI:

10.1080/14680629.2017.1307263

Publication date:

2018

Document Version

Peer reviewed version

Link back to DTU Orbit

Citation (APA):

Madsen, S. S., \& Levenberg, E. (2018). Dynamic backcalculation with different load-time histories. Road Materials and Pavement Design, 19(6), 1314-1333. https://doi.org/10.1080/14680629.2017.1307263

\section{General rights}

Copyright and moral rights for the publications made accessible in the public portal are retained by the authors and/or other copyright owners and it is a condition of accessing publications that users recognise and abide by the legal requirements associated with these rights.

- Users may download and print one copy of any publication from the public portal for the purpose of private study or research.

- You may not further distribute the material or use it for any profit-making activity or commercial gain

- You may freely distribute the URL identifying the publication in the public portal

If you believe that this document breaches copyright please contact us providing details, and we will remove access to the work immediately and investigate your claim. 


\title{
Dynamic backcalculation with different load-time histories
}

\author{
Stine Skov Madsen ${ }^{1}$, Eyal Levenberg ${ }^{2}$ \\ ${ }^{1}$ Solid Mechanics, Department of Mechanical Engineering, Technical University of Denmark \\ ${ }^{2}$ Geotechnics and geology, Department of Civil Engineering, Technical University of Denmark
}

\begin{abstract}
This paper focused attention to the Falling Weight Deflectometer (FWD) load-time history. For a commonly used device, it studied the pulse generation mechanism and the influence of different load histories on backcalculation results. In this connection, a semi-analytic impact theory was first introduced for realistically simulating FWD pulse generation. Then a newly developed finite element code was presented for FWD interpretation; the code is capable of addressing dynamics, timedependent layer properties, and quasi-nonlinear behavior. Both new developments were demonstrated for an experimental dataset that resulted from operating an FWD with different loading configurations. It was found that backcalculated parameters are sensitive to the FWD pulse features. Consequently, it is recommended that, whenever advanced pavement characterization is sought, experimental attention should be placed on generating diverse FWD pulse histories. Collectively, the resulting deflection histories will contain pertinent constitutive information for supporting the calibration of more complex pavement models.
\end{abstract}

Keywords: Falling weight deflectometer, Finite element method, Dynamic backcalculation, load-time history

\section{Introduction}

The Falling Weight Deflectometer (FWD) is a standard non-destructive testing device for the pavement industry (ASTM D4694-09, 2015). In general terms, an FWD is designed to generate a short load pulse at the pavement surface, of the order of 30 milliseconds in duration, and record the associated surface deflections. This impact type of loading is achieved by dropping a weight from a predetermined height, and the deflections are measured by an array of geophones. In most cases FWD measurements are interpreted with the aim of identifying the in situ mechanical properties of the individual system layers (e.g. Bush and Baladi (1989); Quintus et al. (1994); Tayabji and Lukanen (2000)). This is commonly performed by means of backcalculation, wherein model-generated deflections are best matched against field-measured deflections.

Based on the underlying choice for pavement modelling, backcalculation schemes may differ in complexity (e.g., Ullidtz and Stubstad (1985); Shao et al. (1986); Chatti et al. (2004); Lee (2014)). Traditional procedures, which are still widely employed by engineers, ignore inertia effects, disregard time-dependent layer properties, and focus on matching peak deflections only; most are also limited to linear behaviour and isotropic properties. The more advanced schemes allow for backcalculation of time-dependent properties, consider nonlinear behavior, and account for dynamic effects; these focus on matching entire deflection histories. The large body of research associated with the FWD has mainly dealt with two aspects: (i) forward pavement modelling, and (ii) performing the inverse problem. Rather limited work has addressed the device loading mechanism, i.e., the ability of controlling or regulating the FWD pulse history, and consequently the influence such manipulation has on the interpretation outcomes.

This paper focuses attention to the FWD load-time history; it studies the pulse generation mechanism and attributes, and also the influence of different load histories on backcalculated outcomes. A semi-analytic impact theory is first introduced, potentially capable of simulating FWD pulse histories. Then, the impact theory is applied to the analysis of drop experiments; these included operating an FWD with different loading configurations over a given pavement. Presented next is a new finite element (FE) code for forward-modelling the response of pavements to FWD impact. This new code is capable of addressing dynamics, time-dependent layer properties, and variation of properties with depth (i.e., pseudo-nonlinear behaviour). Lastly,

Corresponding author: Stine Skov Madsen, Email: sskk@mek.dtu.dk 
the FE code is employed to backcalculate the measured deflections from the drop experiments, and investigate the effects of the different loading histories on the inferred layer properties. The specific investigation results are presented and discussed, from which some general conclusions are offered.

\section{Impact analysis}

\subsection{Semi-analytic model}

A simplified analytic model for FWD impact was offered by Sebaaly et al. (1985). Their work considered (see Figure 1(a)) a mass $m$ with an underlying weightless buffer that is dropped from a certain height; the buffer was represented by a linear spring with stiffness $k$. In this case the governing differential equation is simply: $m \ddot{x}+k x=m g$ wherein $g$ is the gravitational acceleration of the earth, $x$ denotes the vertical position of the mass at time $t$, and ( $\left.{ }^{(}\right)$indicates differentiation with respect to time. The solution for this equation commences when contact is first made with the ground and the spring begins to compress. The initial conditions are therefore $x(0)=0$ and $\dot{x}(0)=\sqrt{2 g x_{0}}$ wherein $x_{0}$ is the drop height. If the spring remains 'glued' to the pavement surface after impact, the load-time history applied to the pavement $F(t)=k x(t)$ can be expressed analytically as follows

$$
F(t)=\sqrt{2 m g x_{0} k} \sin (t \sqrt{k / m})-m g \cos (t \sqrt{k / m})+m g
$$

The shape of this load-time history is approximately a haversine (i.e. symmetric), with a 'first pulse' duration $\Delta T_{1}$ given by

$$
\Delta T_{1}=\sqrt{\frac{m}{k}}\left(\arctan \left(\frac{2 \sqrt{2 k x_{0} m g}}{2 k x_{0}-m g}\right)+\pi\right)
$$

A typical FWD configuration drops masses in the range of 100 to $400 \mathrm{~kg}$ from heights in the range of 0.05 to $0.40 \mathrm{~m}$. Based on the above expressions, and considering $k=10^{6} \mathrm{~N} / \mathrm{m}$, two possible cases are graphically illustrated in Figure 1; one where a single mass is dropped from two different heights (Figure 1(b)), and another where two different masses are dropped from the same height (Figure 1(c)). In the first case, where $m=100 \mathrm{~kg}$, it can be seen that if $x_{0}$ is increased from 0.05 to $0.40 \mathrm{~m}$ the first pulse duration varies only marginally, between 33.4 and $32.1 \mathrm{~ms}$. On the other hand, the peak load varies considerably between 10.9 to $29.0 \mathrm{kN}$. In the second case, where $x_{0}=0.20 \mathrm{~m}$, it can be seen that if $m$ is increased from 100 to $200 \mathrm{~kg}$ the first pulse duration varies considerably, from 32.4 to $46.4 \mathrm{~ms}$. At the same time, the peak load also varies, from 20.8 to $30.0 \mathrm{kN}$. It can therefore be concluded that for a given mass, adjusting the drop height provides some control over the peak load without influencing the pulse duration. Alternatively, by changing the mass different pulse durations can be produced for different peak loads. Effects of changes in the buffer system (i.e. different $k$-values) are not displayed in Figure 1 . In essence, for a given mass and drop height, an increase in $k$ translates into a shorter pulse duration and higher peak load (and vice versa).
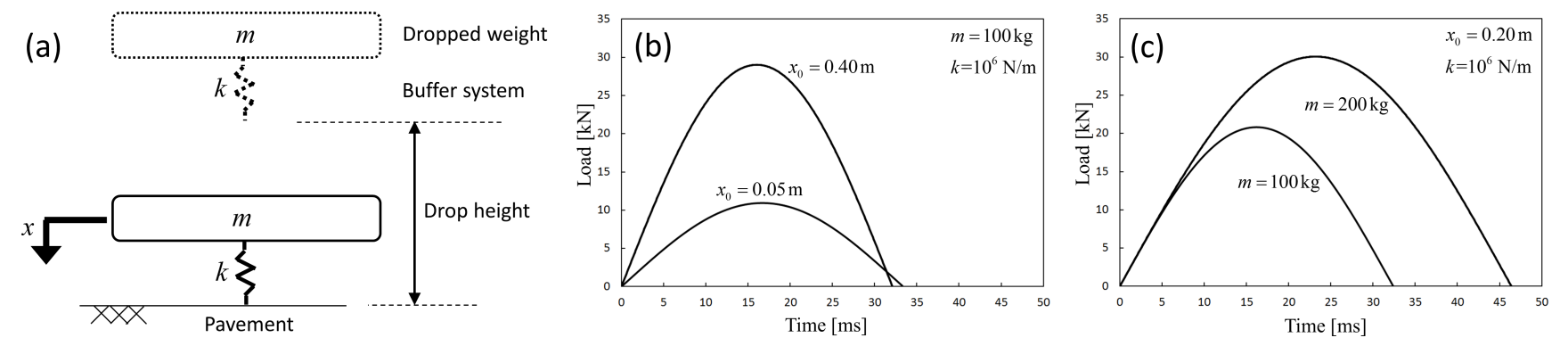

Figure 1: Simplified FWD loading model; (a) spring-mass analog, (b) sensitivity to drop height, and (c) sensitivity to dropped weight.

Generation of FWD load-time history was investigated further by Lukanen (1992). His work dealt with different buffer designs, and experimentally demonstrated that load pulses do not approximate a haversine shape. In actuality, the rate of load rise was higher compared with the unloading rate, and very often some undulation or ripple appeared before the peak. Changing the buffer cross-sectional design (flat, rounded, or semi-rounded), was also demonstrated to influence the pulse shape. These effects can neither be captured nor reproduced with the idealized loading model in Figure 1(a) and the associated formulation given by equations (1) and (2). 
As means of capturing the aforementioned effects, a refined and more realistic FWD loading model is hereafter proposed. The model is shown in Figure 2; it is based on a typical and popular FWD device configuration (specifically, Dynatest 8000). Unlike the simplified model in Figure 1(a), three separate masses are included, wherein $m_{1}$ represents the dropped mass, $m_{2}$ represents the hit bracket, and $m_{3}$ represents the loading plate. In a typical FWD operation, the three masses are first placed quasi-statically on the pavement, then, $m_{1}$ is elevated and allowed to free-fall. The drop height $\left(x_{0}\right)$ measures the initial vertical distance between the surface of the hit bracket $m_{2}$ and the buffer system underlying $m_{1}$. The latter system is considered weightless, represented by a spring and dashpot in parallel. The dashpot simulates simple Newtonian damping with viscosity $c_{1}$ while the spring is chosen as nonlinear-hardening: $k_{1}^{n l}=k_{11}+k_{12} x^{2}$. Physically, the buffer system is composed of several rubber elements, collectively designed to serve as a bumper and absorb the falling mass impact. The chosen model is deemed suitable for this purpose given that it is capable of simulating rubber time-dependency and overall capability for energy absorption. The hardening feature is introduced for capturing effects of non-flat buffer-head geometries and effects of large compressive deformations. Upon impact, the buffer diameter and contact area continually increases (see Mankovits et al., (2014)), which translates into growth of the spring constant value.

The FWD load-cell, which records the applied load-time history, is represented by a massless linear spring with constant $k_{2}$. As can be seen in the figure, it is positioned between $m_{2}$ and $m_{3}$ to measure the load transferred between the two. While this is not the load directly applied to the pavement surface, the associated error is relatively small because $k_{2}$ represents large stiffness. The load-cell mass is included in both $m_{2}$ and $m_{3}$ (split between the two). The final model component is the Viton rubber that resides between the load plate and the pavement surface. It is mainly introduced to ensure full contact across the loaded area between the perfectly flat metallic load plate and the tested surface (which is never perfectly flat), and also to promote the generation of a uniform stress distribution (Uzan and Lytton, 1990). This Viton rubber is represented by a single Kelvin-Voigt viscoelastic element with a spring constant $k_{3}$ and dashpot viscosity $c_{3}$. In this connection, it is important to note that the bottom of the Viton is taken as perfectly rigid. This assumption is incorrect given that the pavement deforms due to the loading. Consequently, the Kelvin-Voigt element properties can be viewed to represent some combination of the Viton properties and the pavement properties.

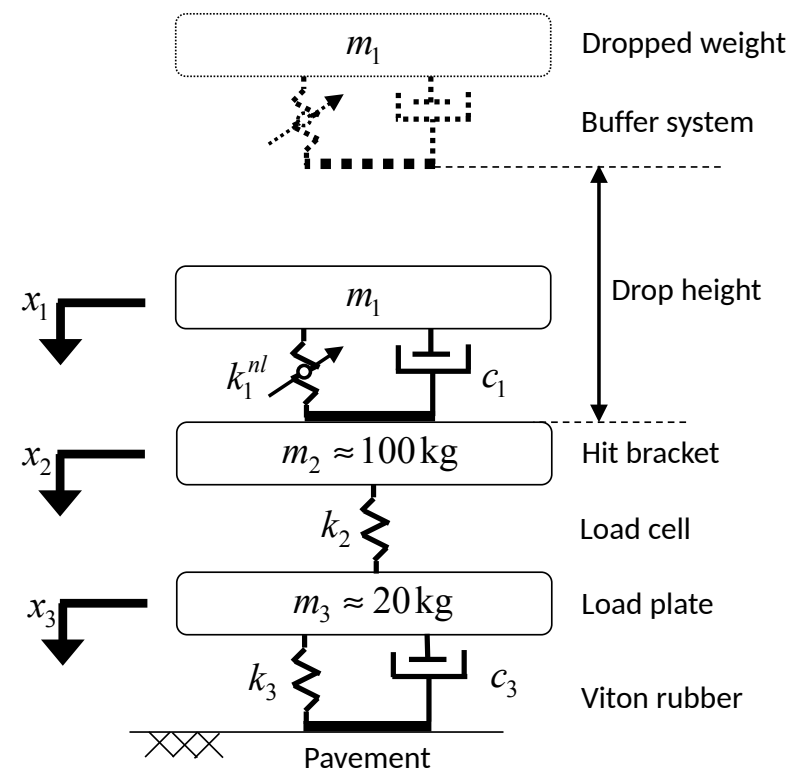

Figure 2: Realistic FWD loading model.

The following nonlinear set of three differential equations (3)-(5) is associated with the FWD model shown in Figure 2. Each equation represents dynamic equilibrium over one of the masses. The formulation is based on the premise that the Viton rubber does not detach from the pavement. On the other hand, allowance is given in the formulation for $m_{1}$ to bounce off and separate from $m_{2}$. For this purpose the Macaulay brackets (defined as $\langle x\rangle=0.5 x+0.5|x|$ ) are introduced to mathematically 
prevent tension from taking place between the two masses:

$$
\begin{aligned}
& m_{1} g=m_{1} \ddot{x}_{1}+\left\langle c_{1}\left(\dot{x}_{1}-\dot{x}_{2}\right)+k_{11}\left(x_{1}-x_{2}\right)+k_{12}\left(x_{1}-x_{2}\right)^{3}\right\rangle \\
& \left\langle c_{1}\left(\dot{x}_{1}-\dot{x}_{2}\right)+k_{11}\left(x_{1}-x_{2}\right)+k_{12}\left(x_{1}-x_{2}\right)^{3}\right\rangle=m_{2} \ddot{x}_{2}+k_{2}\left(x_{2}-x_{3}\right) \\
& k_{2}\left(x_{2}-x_{3}\right)=m_{3} \ddot{x}_{3}+c_{3} \dot{x}_{3}+k_{3} x_{3}
\end{aligned}
$$

When solving these equations, analysis commences at the instant the buffer system makes first contact with the hit bracket $\left(m_{2}\right)$. The associated initial conditions in this case are: $x_{1}(0)=x_{2}(0)=x_{3}(0)=0, \dot{x}_{2}(0)=\dot{x}_{3}(0)=0$, and $\dot{x}_{1}(0)=\sqrt{2 x_{0} g}$. In the following section this model is applied to investigate experimentally measured load-time histories.

\subsection{Experimental}

FWD drop experiments took place inside a $50 \mathrm{~m} \times 50 \mathrm{~m}$ hangar isolated from outside environment. The hangar floor was composed of a cast-in-place concrete with an average thickness of $150 \mathrm{~mm}$. Below the concrete was a $200 \mathrm{~mm}$ layer of uniformly graded, round gravel overlaying a typical Danish glacial bolder clay with granite inclusions of up to $100 \mathrm{~mm}$ in size. Based on nearby bore logs, the bolder clay at the site extends to a depth of about $8 \mathrm{~m}$ and the water table level is deeper than $20 \mathrm{~m}$. In the end of 2014 a portion of the concrete floor was replaced with a new asphalt pavement. A rectangular cut of $5 \mathrm{~m} \times 3 \mathrm{~m}$ in size was performed and the underlying materials were excavated to a depth of $550 \mathrm{~mm}$. The bottom was compacted with vibratory rollers to serve as formation level for a $520 \mathrm{~mm}$ pavement structure consisting of $400 \mathrm{~mm}$ of crushed granular base and $120 \mathrm{~mm}$ of asphalt concrete (AC). The base was compacted in several lifts using a Jumping Jack compactor and the asphalt layer was compacted in two lifts by a double-drum steel-wheeled roller and a vibratory plate. The AC mixture consisted of a combination of crushed and uncrushed materials with a maximum aggregate size of $22.4 \mathrm{~mm}$, and a relatively soft bitumen graded as 70/100 (EN 12591:2009). Figure 3 graphically presents the above description; Figure 3(a) presents a plan view of the testing area and Figure 3(b) presents a cross-sectional view. As may be seen, the surface of the asphalt pavement was slightly lower than the surrounding concrete floor allowing for a future addition of a $30 \mathrm{~mm}$ AC lift.

In the end of 2015 (i.e., about a year after construction), the asphalt pavement was tested by an FWD. The specific device used was a Dynatest model 8012 which is capable of faster operation as compared to traditional systems, but is essentially identical to standard devices for all practical reasons. The load plate was positioned as seen in Figure 3(a) along with eight geophones placed at the following offset distances (from the center): 0, 200, 300, 600, 900, 1200, 1500 and $1800 \mathrm{~mm}$. The load plate was $300 \mathrm{~mm}$ in diameter; it was segmented into four parts and included a $6 \mathrm{~mm}$ Viton rubber placed underneath it. The buffers used for the testing were hard cylinder buffers (Shore hardness of 80A), slightly rounded at the tip having a 110 $\mathrm{mm}$ diameter and a height of $84 \mathrm{~mm}$.

A total of 24 FWD drops were executed, spanning the full operational range of the device in terms of drop height, drop weight, and number of buffers. All drops were executed within a period of 0.5 hours, during which the AC temperature was $22^{\circ} \mathrm{C}$. Eight separate FWD tests out of the 24 were selected for further analysis in this study (numbered sequentially for convenience). Their load-time histories are presented by the dotted lines in Figure 4 while the associated operational settings and load-time history features are listed in Table 1. Figure 4(a) shows three tests for which only the drop height differed. As can be seen, for a given buffer configuration and drop mass, increasing the drop height generates an increase in the peak load and a shortening of the pulse length (i.e. earlier peak occurrence). Figure 4(b) shows three drops for which the drop height and the buffer arrangement were kept constant, but the dropped mass differed. In this case, as the mass was increased, both the peak load and the pulse duration increased (i.e. later peak occurrence). Figure 4(c) includes three tests that exhibited nearly identical peaks but different pulse durations. These drops cannot be generated by adjusting or varying only one single device option.

Also included in Figure 4 (as solid lines) are computed load-time histories. These were generated from numerically solving the set of Equations (3),(4) and (5) within the time interval $t=0$ to $t=0.7 \mathrm{~s}$ by a Runge-Kutta-Fehlberg method (fourth-fifth order). The employed model parameters are given in Table 2. These values were obtained from best fitting the measured FWD pulses across the different test configurations. A fitting approach was needed because the realistic FWD model is an idealization, and other device components or mechanical connections may influence the pulse generation (one example is friction with the external frame that connects the three masses to the FWD trailer). As expected, the parameters $k_{11}, k_{12}$ and $c_{1}$ change based on the number of buffers used. The values of $k_{11}$ and $k_{12}$ grow with the increase in the number of buffers while the damping decreases. These trends are to be expected, yet they are not linear with the number of buffers. The values of $k_{11}$ appear reasonable, of the order of $10^{6} \mathrm{kN} / \mathrm{m}$ (Sebaaly et al., (1985)). The parameters $k_{2}, k_{3}$ and $c_{3}$ are common to all cases 

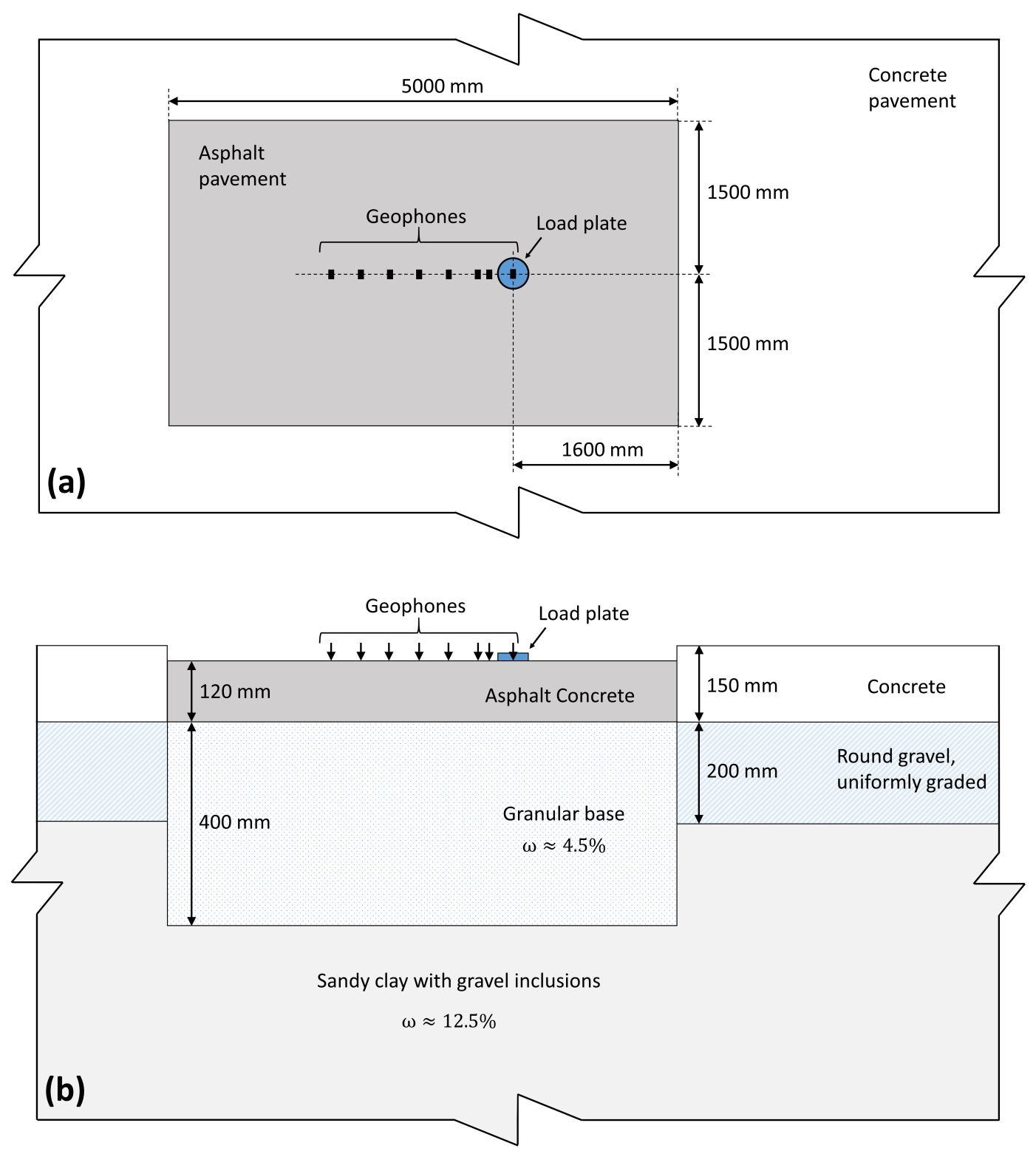

Figure 3: FWD testing area: (a) plan view, and (b) cross-sectional view.

and therefore are not affected when the buffer system is manipulated. The load-cell stiffness $k_{2}$ converged to a value that is a lower than expected (one would expect about $10^{9} \mathrm{kN} / \mathrm{m}$ ), possibly due to some additional flexibility in the device. There is no reference for $k_{3}$ and $c_{3}$ given that they include the properties of the tested pavement. As can be graphically seen, the model is able to reproduce an FWDs load-time history for any number of buffers and for a range of drop heights and weights. Peak load levels are adequately replicated as well as the overall asymmetry in pulse shape; the model was also able to simulate the undulation of the load-time history (in contrast to the charts in Figure 1). Within the device capability range, such a calibrated model at hand can serve as guide to FWD operators in generating predefined load-time histories.

In the beginning of 2016, well after all FWD tests were concluded, the asphalt pavement section was further investigated to collect more geotechnical characteristics. The investigation reaffirmed the earlier bore log findings and the system layering. It also included vane testing which displayed a trend of increasing shear resistance with depth, from $125 \mathrm{kN} / \mathrm{m}^{2}$ close to the subgrade surface to $330 \mathrm{kN} / \mathrm{m}^{2}$ at a depth of $4 \mathrm{~m}$. Moreover, an AC core was extracted from the pavement for subsequent 

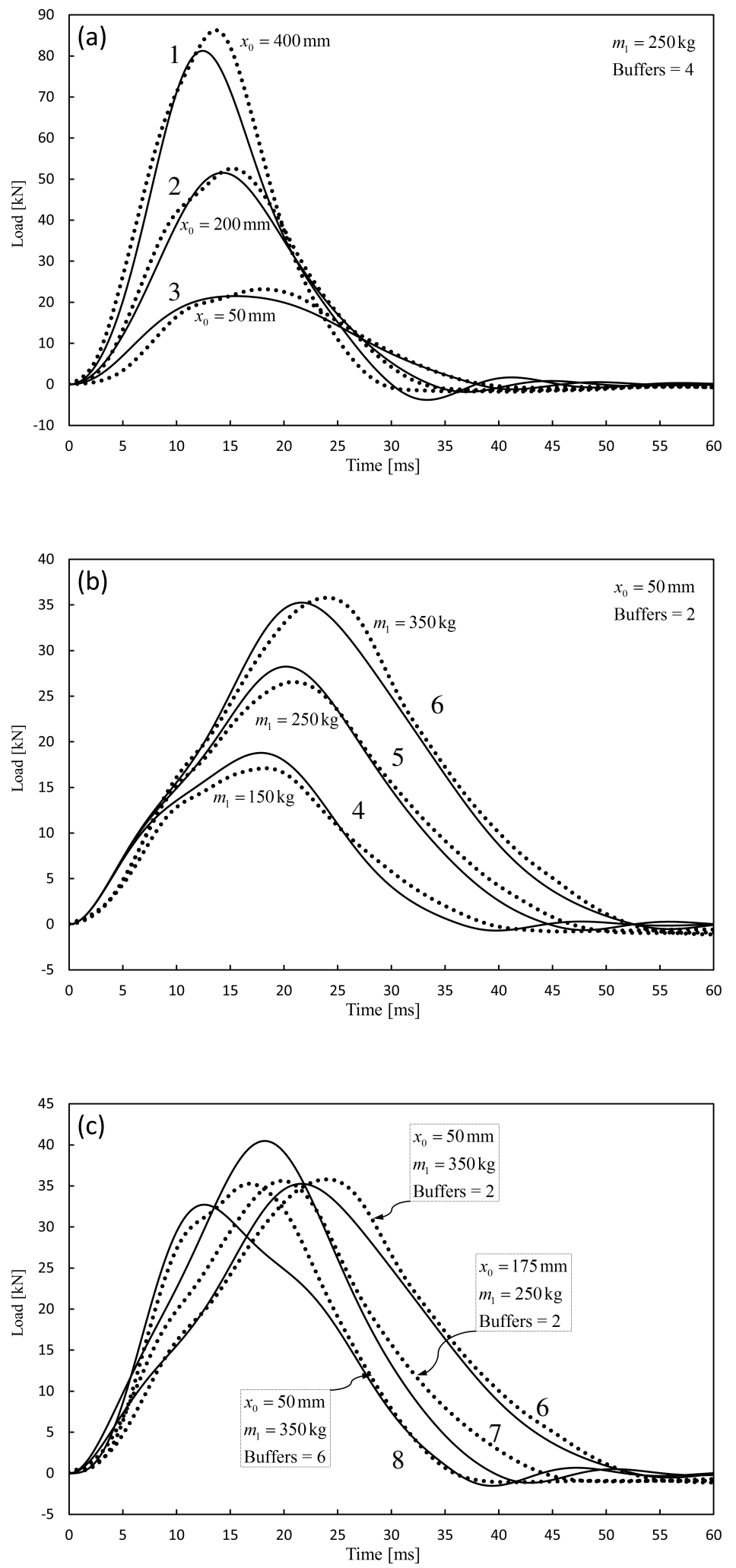

Figure 4: Measured (dotted line) and modelled (solid line) FWD load-time histories 


\begin{tabular}{l|c|c|c|c|c} 
Test & Buffers & Drop mass $[\mathrm{kg}]$ & Drop height $[\mathrm{mm}]$ & Pulse duration $[\mathrm{ms}]$ & Peak load $[\mathrm{kN}]$ \\
\hline 1 & 4 & 250 & 400 & 29.5 & 86 \\
2 & 4 & 250 & 200 & 33.0 & 52 \\
3 & 4 & 250 & 50 & 37.0 & 23 \\
4 & 2 & 150 & 100 & 39.0 & 17 \\
5 & 2 & 250 & 100 & 47.5 & 26 \\
6 & 2 & 350 & 100 & 52.0 & 35 \\
7 & 2 & 250 & 175 & 44.0 & 35 \\
8 & 6 & 350 & 50 & 35.5 & 35
\end{tabular}

Table 1: Selected FWD drop experiments: pulse features and device operational settings.

\begin{tabular}{c|c|c|c} 
& \multicolumn{3}{|c}{ Number of buffers } \\
\hline Model element & 2 & 4 & 6 \\
\hline$k_{11}[\mathrm{~N} / \mathrm{m}]$ & $5.495 \cdot 10^{5}$ & $1.259 \cdot 10^{6}$ & $2.239 \cdot 10^{6}$ \\
\hline$k_{12}\left[\mathrm{~N} / \mathrm{m}^{3}\right]$ & $5.129 \cdot 10^{9}$ & $1.072 \cdot 10^{10}$ & $2.692 \cdot 10^{10}$ \\
\hline$c_{1}[\mathrm{Ns} / \mathrm{m}]$ & $3.802 \cdot 10^{3}$ & $2.692 \cdot 10^{3}$ & $9.333 \cdot 10^{1}$ \\
\hline$k_{2}[\mathrm{~N} / \mathrm{m}]$ & \multicolumn{3}{|c}{$1.622 \cdot 10^{7}$} \\
\hline$k_{3}[\mathrm{~N} / \mathrm{m}]$ & $3.090 \cdot 10^{3}$ \\
\hline$c_{3}[\mathrm{Ns} / \mathrm{m}]$ & \multicolumn{3}{|c}{$8.318 \cdot 10^{4}$}
\end{tabular}

Table 2: Calibrated values for the realistic FWD loading model in Figure 2.

linear viscoelastic characterization. Tests were performed in indirect tension mode under a constant temperature of $20^{\circ} \mathrm{C}$. They were analysed assuming a constant Poisson's ratio of 0.35 according to the methodology proposed in Levenberg and Michaeli (2013). The experimental information obtained from this geotechnical investigation is utilized hereafter for guiding some modelling choices, and later on for evaluating backcalculation results.

\section{Pavement modelling}

This section is concerned with the forward modelling of an FWD experiment for subsequent inverse analysis. The aforementioned pavement was represented as a fully bonded three layer system as shown in Figure 5. In general terms, each layer was assumed to be a linear viscoelastic solid governed by a Kelvin-Voigt type of constitutive relation

$$
\sigma=C \varepsilon+\eta C \dot{\varepsilon}
$$

where $\sigma$ is the stress tensor, $\boldsymbol{\varepsilon}$ and $\dot{\boldsymbol{\varepsilon}}$ are the strain and strain-rate tensors, respectively, $\boldsymbol{C}$ is the constitutive tensor and $\eta$ denotes material damping. From a modeling point of view the benefit of this choice, and in particular the introduction of $\eta$, is the ability to simulate deflection shapes and timing delays that cannot be solely reproduced with elastodynamics. $\eta$ has a small influence on the deflection magnitudes and a smoothing effect on the time-history curves. From a physical point of view, AC layers are time-dependent and should therefore be modeled as such. Also, soils and unbound granular materials have been reported to exhibit time-dependent behavior (e.g. Augustesen et al. (2004); Liingaard et al. (2004); Zou et al. (2012); Levenberg (2014); Seed et al. (1986)).

For small strains, $\boldsymbol{\varepsilon}=\boldsymbol{\nabla} \mathbf{u}+(\boldsymbol{\nabla u})^{T}$ where $\mathbf{u}$ is the displacement vector and $\boldsymbol{\nabla}$ is the gradient operator. Assuming isotropy, the constitutive tensor is governed by two parameters: Young's modulus $E$ and Poisson's ratio $v$. In Figure 5, the material properties are identified for each layer by subscripts 1,2 and 3 referring to AC layer, base layer, and subgrade, respectively. Given that $\mathrm{AC}$ is a thermosensitive material, temperature gradients may be taken into consideration via sub-layering in combination with 


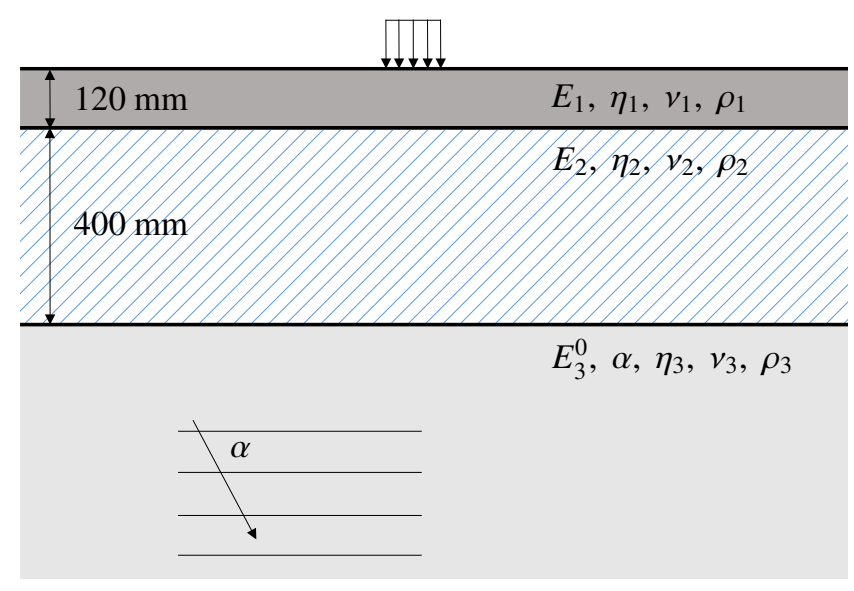

Figure 5: Three layered model of the experimental pavement in Figure 3.

appropriate geophone spacing. For the current case, wherein the AC layer is relatively thin and the pavement is located indoors, such modelling and experimental consideration was assumed unneeded.

The modulus of the subgrade layer was allowed to vary as a function of depth; an expression suggested by Ullidtz (1998) was employed in this connection

$$
E_{3}(z)=E_{3}^{0}\left(\frac{z}{z_{0}}\right)^{\alpha}
$$

where $E_{3}^{0}$ is Young's modulus at the top of the subgrade, $z_{0}$ is a reference depth, and $\alpha$ is a unitless exponent governing the rate of modulus increase or decrease. For the pavement considered in this work the reference depth is $z_{0}=520 \mathrm{~mm}$ (see Figure 5), and based on the vane test results, $\alpha$ was assumed positive. From a modeling point of view, an increase in $\alpha$ diminishes the deflection magnitudes, with slight effect on the shape features.

The general equation of motion governing elastic wave propagation in a continuum is given by e.g. Cook et al. (2002)

$$
\left(\nabla^{T} \sigma\right)^{T}=\rho \frac{\partial^{2} \mathbf{u}}{\partial t^{2}}
$$

where $\rho$ is the mass density. The system is assumed stress free until the surface of the top layer is exposed to an FWD stress history $\sigma(t)$ uniformly spread over a circle with radius $r_{0}=150 \mathrm{~mm}$. To solve for the resulting dynamic response, the FE approach was employed. For this purpose the equation of motion is multiplied by a virtual displacement field $\tilde{\mathbf{u}}$ followed by integration over the volume and reformulation using the divergence theorem yielding

$$
\int_{S} \tilde{\mathbf{u}}^{T} \boldsymbol{\sigma} \mathbf{n}_{\mathbf{z}} d S-\int_{V}\left[\tilde{\boldsymbol{\varepsilon}}^{T} \boldsymbol{\sigma}+\rho \tilde{\mathbf{u}}^{T} \ddot{\mathbf{u}}\right] d V=0
$$

wherein $S$ and $V$ denote integration over surface area and volume, respectively, and $\mathbf{n}_{\mathbf{z}}$ is a unit vector indicating the vertical direction of load. The spatial variation of the actual and the virtual displacement fields are represented by shape functions via the relations

$$
\begin{aligned}
& \mathbf{u}=\mathbf{N} \mathbf{d} \\
& \tilde{\mathbf{u}}=\tilde{\mathbf{N}} \tilde{\mathbf{d}}
\end{aligned}
$$

with $\mathbf{d}$ as the nodal displacements and the shape function matrices $\mathbf{N}$ or $\tilde{\mathbf{N}}$ in the form

$$
\mathbf{N}=\left[\begin{array}{ccccccc}
N_{1} & 0 & N_{2} & 0 & \cdots & N_{n} & 0 \\
0 & N_{1} & 0 & N_{2} & \cdots & 0 & N_{n}
\end{array}\right]
$$


Insertion of the shape functions into Equation (9) yields the wave equation in matrix form

$$
\mathbf{M u ̈}+\mathbf{Z} \dot{\mathbf{u}}+\mathbf{K u}=\mathbf{F}(t)
$$

in which $\mathbf{M}$ is the mass matrix, $\mathbf{Z}$ is the damping matrix, $\mathbf{K}$ is the stiffness matrix, $\mathbf{F}(t)$ is the load vector, and $\mathbf{u}$ refers to nodal displacements.

\subsection{Numerical implementation}

Since the force is circular and uniformly distributed, and all other model components exhibit rotational symmetry about the load axis, the problem is treated as axisymmetric with radial coordinate $r$, axis of revolution $z$ and circumferential coordinate $\theta$. In this case the displacement field becomes a function of the radial displacement $u_{r}$ and the axial displacement $u_{z}$ only, i.e. $\mathbf{u}=\left[u_{r}, u_{z}\right]^{T}$. The strain-displacement relationships are

$$
\varepsilon_{r}=\frac{\partial u_{r}}{\partial r} \quad \varepsilon_{\theta}=\frac{u}{r} \quad \varepsilon_{z}=\frac{\partial u_{z}}{\partial z} \quad \gamma_{r z}=\frac{\partial u_{r}}{\partial z}+\frac{\partial u_{z}}{\partial r}
$$

In array format the strain and corresponding stress are written as

$$
\begin{aligned}
\boldsymbol{\sigma} & =\left[\sigma_{r r}, \sigma_{\theta \theta}, \sigma_{z z}, \sigma_{r z}\right]^{T} \\
\boldsymbol{\varepsilon}=\boldsymbol{\partial} \mathbf{u} & =\left[\varepsilon_{r r}, \varepsilon_{\theta \theta}, \varepsilon_{z z}, \gamma_{r z}\right]^{T}
\end{aligned}
$$

The strain-displacement operator is expressed by

$$
\boldsymbol{\partial}=\left[\begin{array}{cc}
\partial / \partial r & 0 \\
1 / r & 0 \\
0 & \partial / \partial z \\
\partial / \partial z & \partial / \partial r
\end{array}\right]
$$

Since no variation occurs in the circumferential direction, the volume $d V$ of an element can be expressed as

$$
d V=2 \pi r d A
$$

where $d A$ is the cross-sectional area of an element. Similarly, the element of surface $d S$ can be expressed as

$$
d S=2 \pi r d s
$$

where $d s$ denotes an element length. Insertion of (18) and (19) into (9) defines the matrices in (13) as

$$
\begin{aligned}
\mathbf{M} & =2 \pi \int_{A} \rho \tilde{\mathbf{N}}^{T} \mathbf{N} r d r d z \\
\mathbf{Z} & =\eta 2 \pi \int_{A} \tilde{\mathbf{B}}^{T} \mathbf{C B} r d r d z \\
\mathbf{K} & =2 \pi \int_{A} \tilde{\mathbf{B}}^{T} \mathbf{C B} r d r d z
\end{aligned}
$$

where $\mathbf{B}=\boldsymbol{\partial} \mathbf{N}$ denotes the strain displacement matrix and the constitutive matrix $\mathbf{C}$ for layer $i$ is given by

$$
\mathbf{C}=\frac{E_{i}}{\left(1+v_{i}\right)\left(1-2 v_{i}\right)}\left[\begin{array}{cccc}
1-v_{i} & v_{i} & v_{i} & 0 \\
v_{i} & 1-v_{i} & v_{i} & 0 \\
v_{i} & v_{i} & 1-v_{i} & 0 \\
0 & 0 & 0 & \left(1-2 v_{i}\right) / 2
\end{array}\right]
$$

Finally, the load vector in Equation (13) is given by

$$
\mathbf{F}(t)=2 \pi \int_{r_{0}} \boldsymbol{\sigma}(t) \boldsymbol{n}_{z} r d s
$$




\subsection{Mesh generation}

This section presents a method for generating a finite element mesh that balances computational time and numerical accuracy. For the problem at hand, waves generated at the surface propagate into the medium while decaying with increasing distance from the source. Accordingly, an efficient mesh capturing this behaviour consists of placing the smallest elements near the load center and then placing larger and larger elements as distance increases. Such a domain discretization approach is presented in Figure 6(a).

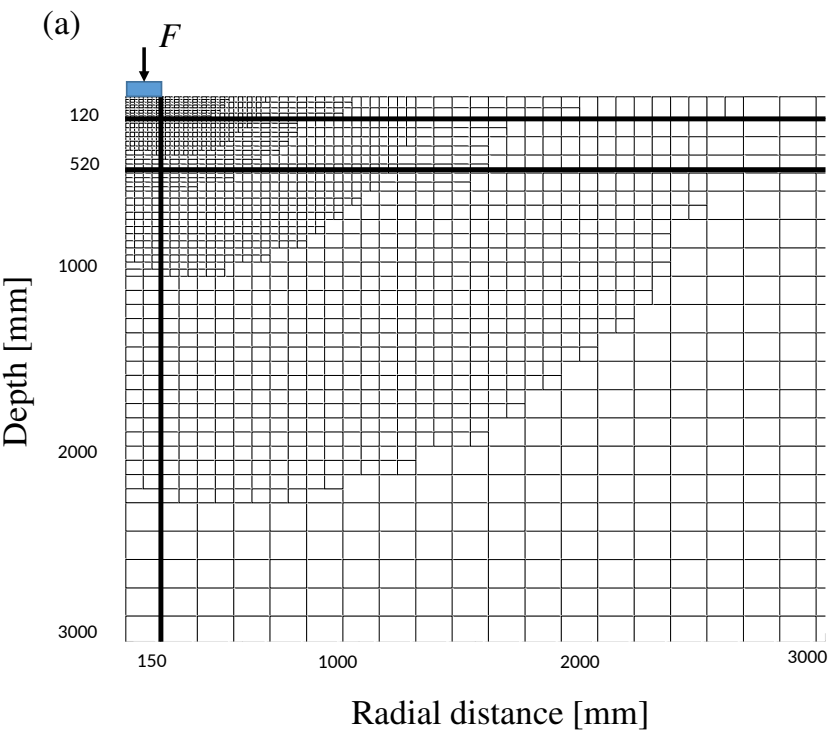

(b)

Figure 6: FE mesh: (a) illustration of elements increasing in size with distance from loading area, and (b) chart of mesh generation parameters.

More specifically, the mesh generation strategy devised herein was governed by four user-selected parameters: a minimum element size $\Delta x_{\min }$, a maximum element size $\Delta x_{\max }$, a growth rate parameter $G_{R}$, and an overall domain size $\bar{d}_{0}$. From an initial (minimum) element size $\Delta x_{\min }$ the elements double in size as a function of their distance from the load center, $\bar{d}$, until reaching a maximum element size $\Delta x_{\max }$. From this point onward, until the domain boundary $\bar{d}_{0}$ is approached, the size of all elements remain $\Delta x_{\max }$. In this scheme the $G_{R}$ parameter controls the 'rate' at which element size is doubled. All four mesh controlling parameters are illustrated graphically in Figure 6(b) which depicts element side length $\Delta x$ versus distance $\bar{d}$. Additional restrictions in the mesh generation, not presented in the figure, were applied to ensure that none of the elements cross the interface between two adjacent layers and that the load is distributed over an integer number of elements.

The overall domain size $\bar{d}_{0}$ was chosen to be large enough such that the waves generated at the load cannot reach the boundary during the analysis period. A domain size $\bar{d}_{0}=9500 \mathrm{~mm}$ was found adequate in this respect given that an FWD drop test is a relatively short-lasting event, of the order of $80 \mathrm{~ms}$ in duration. The other mesh parameters were selected such that computed deflections are accurate to within $\pm 1 \mu \mathrm{m}$ which is equivalent to the level of deflection accuracy measured by a typical FWD device. Accordingly, the final generation values were: $\Delta x_{\min }=19 \mathrm{~mm}, \Delta x_{\max }=625 \mathrm{~mm}$, and $G_{R}=0.06$.

A numerical study was carried out in an attempt to quantify any side effects originating from the existence of a concrete pavement surrounding the test section. The axisymmetric analysis was also employed for this purpose. It included positioning a concrete plate in the model, from a redial distance of $1.5 \mathrm{~m}$ and extending towards the boundary of the mesh. An FWD drop was simulated and the computed deflections histories were compared to the case shown in Figure 5. Observed differences were smaller than $\pm 1 \mu \mathrm{m}$ and therefore considered negligibly small and inconsequential.

\section{Backcalculation}

Backcalculation was employed here to best estimate the constitutive parameters of the pavement layers in Figure 5 under the different loading conditions in Figure 4. For this purpose the measured load histories were used as an input to the numerical formulation for generating model deflections. Applied load was discretized with a constant interval of $0.5 \mathrm{~ms}$ into 60-120 time steps depending on the load pulse duration. The level of mismatch between the modelled and measured deflections 


\begin{tabular}{l|c|c|c|c|c} 
Layer & $v[-]$ & $\rho\left[\mathrm{kg} / \mathrm{m}^{3}\right]$ & $E[\mathrm{MPa}]$ & $\eta[\mathrm{ms}]$ & $\alpha[-]$ \\
\hline 1 & 0.35 & 2400 & {$[1000-7000]$} & {$[0.05-5.00]$} & - \\
2 & 0.35 & 2000 & {$[150-750]$} & {$[0.05-5.00]$} & - \\
3 & 0.35 & 1800 & {$[25-180]^{*}$} & {$[0.05-5.00]$} & {$[0.0-3.0]$}
\end{tabular}

Table 3: Layer properties of the model pavement. $\rho$ and $v$ are prefixed during backcalculation. $E, \eta$ and $\alpha$ are adjustable during backcalculation within the defined ranges (*range for $E_{3}^{0}$ ).

was subsequently minimized by adjusting the unknown parameters. As shown in Figure 5 there are a total of 13 constitutive parameters that govern the model response. As is commonly accepted, $v_{1}-v_{3}$ and $\rho_{1}-\rho_{3}$, were prefixed before performing the backcalculation; their chosen values are included in Table 3 . The seven remaining parameters: $E_{1}, E_{2}, E_{3}^{0}, \eta_{1}-\eta_{3}$ and $\alpha$ were kept adjustable for the backcalculation algorithm. As means of confining the solution search space, this latter set of sought parameters was bound to within the ranges defined in Table 3. As can be seen, $\eta$ is allowed to vary within a wide range so that the assumption and need to include damping is ultimately determined by the analysis.

\subsection{Minimization approach}

An objective function, $\psi_{a b}$, was defined to express the difference between modelled and measured deflections as follows

$$
\psi_{a b}=\sqrt{\frac{1}{b-a+1} \frac{1}{M} \sum_{k=a}^{b} \sum_{j=1}^{M}\left(D_{k}\left(t_{j}\right)-d_{k}\left(t_{j}\right)\right)^{2}}
$$

where $D_{k}\left(t_{j}\right)$ is the deflection measured by the $k^{t h}$ geophone at discrete times $t_{j}=t_{1}, t_{2}, \ldots, t_{M}$ and $d_{k}\left(t_{j}\right)$ is the corresponding deflection produced by the numerical model. The geophones included in the objective function are $k \in[a ; b]$ where $a$ and $b$ are geophone numbers; $a=1,2, \ldots, 8$ and $b=1,2, \ldots, 8$ with $b \geq a$. As can be seen, $\psi_{a b}$ represents the discrepancy between model and measurement across the entire simulated time for a group of sensors $a, a+1, \ldots, b$ out of the entire available set. The overall goal of the backcalculation process is to minimize $\psi_{18}$ within a total analysis duration $t_{M}$. As means of ensuring

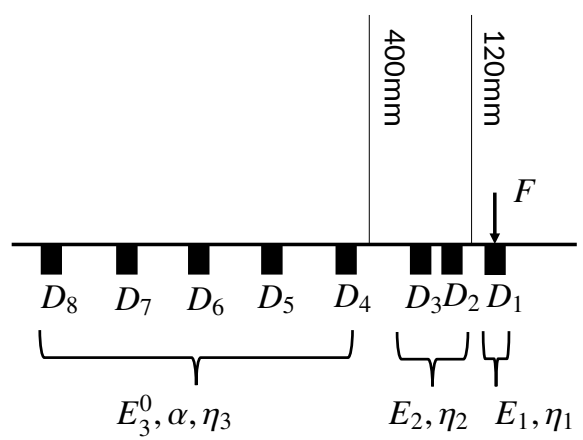

Figure 7: Grouping of geophones based on pavement layering for sequential minimization of $\psi_{18}$ (see Equation (25)).

convergence into physically realistic results $t_{M}$ was chosen to include the first part of the recorded deflections - about $60 \%$. This analysis period covers the deflection rise-time, the peak, and a small portion of the decrease. This truncation is done because deflection recordings are known to be less reliable as time progresses (due to integration of geophone data), producing unrealistic deflection tails (e.g., Uzan, 1994).

Another effort for ensuring convergence into meaningful results involved performing the minimization in stages. Initially, parameter seed values were randomly chosen within the bounds given in Table 3 . Then (Stage I) $\psi_{48}$ was minimized with respect to $E_{3}^{0}, \alpha$ and $\eta_{3}$; in Stage $I I \psi_{23}$ was minimized with respect to $E_{2}$ and $\eta_{2}$; and in Stage $I I I \psi_{1}$ was minimized with respect to $E_{1}$ and $\eta_{1}$. This bottom-up approach is based on an engineering intuition that associates more distant geophone readings with deeper layer properties. The rational for the specific choices in the above stages is illustrated in Figure 7 in which the pavement layering is horizontally superposed over the geophone array configuration. Accordingly, $d_{4}-d_{8}$ were 
deemed more sensitive to the subgrade parameters, $d_{2}-d_{3}$ were deemed more sensitive to the base parameters and $d_{1}$ most closely associated with the AC parameters.

A gradient based method was used for the three stages with gradients calculated using a backward finite difference. In each stage the minimization process was restarted several times with new seed values to broaden the search space for a solution. The optimal (minimal) objective functions in stages I, II and III were denoted $\psi_{48}^{\min }, \psi_{23}^{\min }$ and $\psi_{11}^{\min }$. These entities were employed in the Min-Max sense (Osyczka, 1978) to perform an overall minimization for $\psi_{18}$ with respect to all adjustable parameters (simultaneously). A general purpose unconstrained derivative-free nonlinear optimization algorithm was employed for this final minimization step (Stage IV) (for more details consult Madsen (2016)). A pseudo code for the above described procedure is presented in Algorithm 1.

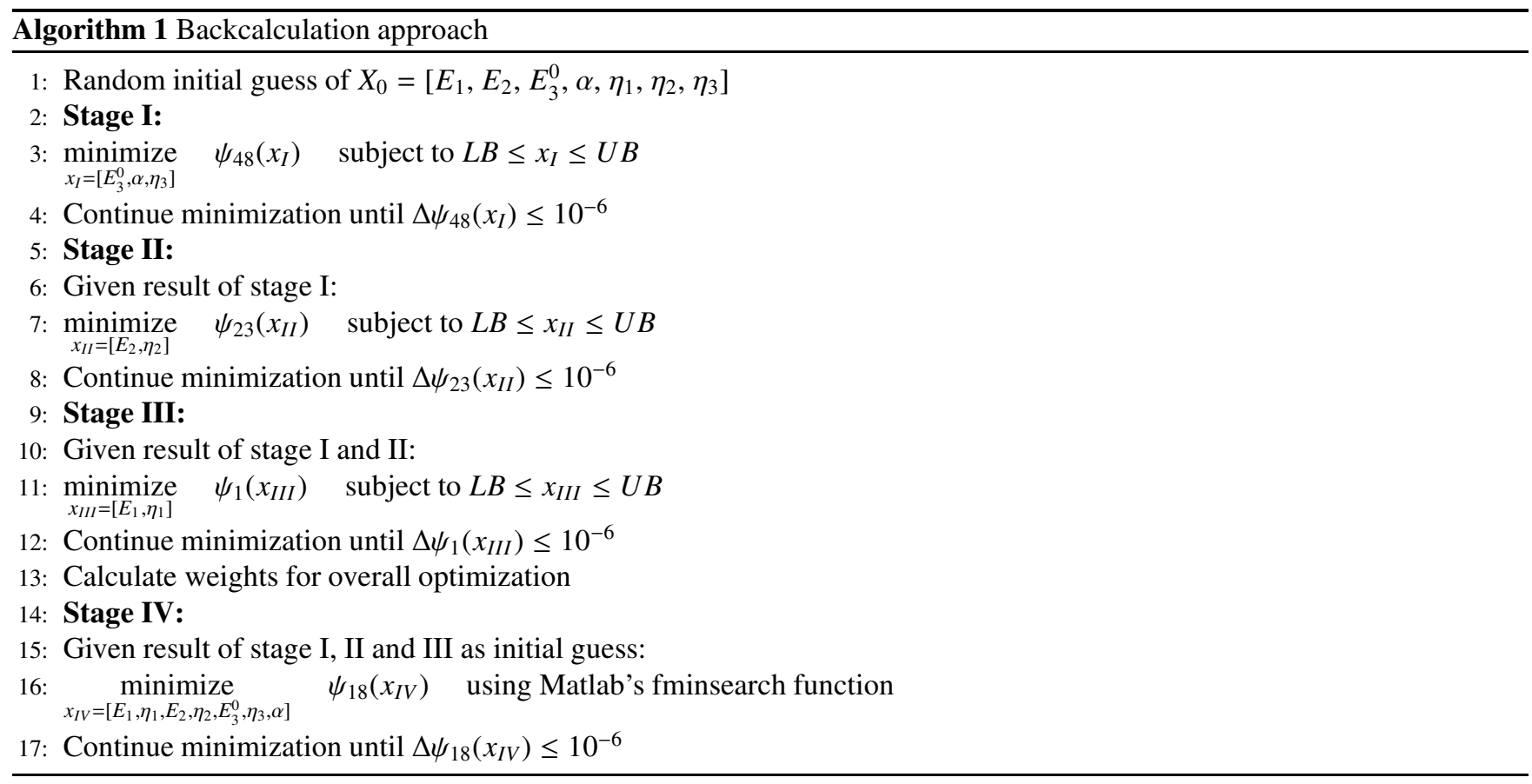

\section{Results}

Included and discussed in this section are backcalculation results. First presented is the fitting obtained between modelled and computed deflection histories for Test 6 and for Test 8. Both drops were similar in terms of peak force but differed with respect to pulse duration (see Figure 4 and Table 1). Geophone measurements and calculated deflections are superposed in Figures 8 and 9. The dashed vertical line indicates the analysis duration in each case (i.e., $t_{M}$ ). As can be graphically seen in both figures, the FE model was able to capture and reproduce both the magnitude and the overall shape of the deflections. Essentially similar charts were obtained for the other tests.

Presented in Table 4 is a summary of the backcalculation results for the eight FWD drops. For the AC layer, $E_{1}$ was found in the range 3065-4561 MPa and $\eta_{1}$ was found in the range of 2.1-4.1 ms. For the base layer, $E_{2}$ was found in the range 246-307 $\mathrm{MPa}$ and $\eta_{2}$ in the range of 0.6-1.1 ms. Finally, for the subgrade, $E_{3}^{0}$ was found in the range 99-153 MPa, $\eta_{3}$ in the range of $0.9-2.0 \mathrm{~ms}$, and $\alpha$ in the range of 1.0-1.4. The latter corresponds well to the vane test results which indicated increasing stiffness profile for the subgrade. Stress-strain Lissajous curves were generated for the average backcalculated base and top-of-subgrade parameters; this was done for a frequency of $30 \mathrm{~Hz}$, i.e., mid of pseudo-frequency range for FWD pulse (see Figure 11(b)). Damping ratios, i.e., quotient of dissipated energy to elastic energy, were directly calculated from resulting elliptical plots. A damping ratio of $8.5 \%$ was found for the base layer and a damping ratio of $13.5 \%$ was found for the upper subgrade. These values fit well within the accepted range (Seed et al. 1986; Bolton and Wilson (1991); Zou et al. (2012)). Across the different analysed cases, the coefficient of variation for the moduli values was relatively small, about $10 \%$, while the 


\begin{tabular}{|c|c|c|c|c|c|c|c|c|}
\hline & \multicolumn{2}{|c|}{ AC properties } & \multicolumn{2}{|c|}{ Base properties } & \multicolumn{3}{|c|}{ Subgrade properties } & \\
\hline Test & $E_{1}[\mathrm{MPa}]$ & $\eta_{1}[\mathrm{~ms}]$ & $E_{2}[\mathrm{MPa}]$ & $\eta_{2}[\mathrm{~ms}]$ & $E_{3}^{0}[\mathrm{MPa}]$ & $\alpha[-]$ & $\eta_{3}[\mathrm{~ms}]$ & $\psi_{18}^{\min }[\mu \mathrm{m}]$ \\
\hline 1 & 4211 & 2.5 & 247 & 0.6 & 99 & 1.2 & 2.0 & 3.6 \\
\hline 2 & 4250 & 2.2 & 238 & 1.1 & 126 & 1.2 & 1.1 & 2.2 \\
\hline 3 & 3786 & 2.8 & 272 & 0.9 & 151 & 1.1 & 0.9 & 1.0 \\
\hline 4 & 3065 & 3.3 & 307 & 0.8 & 129 & 1.4 & 1.3 & 0.8 \\
\hline 5 & 3492 & 4.1 & 285 & 0.8 & 143 & 1.1 & 1.9 & 1.0 \\
\hline 6 & 3953 & 4.5 & 262 & 0.7 & 150 & 1.0 & 2.0 & 1.4 \\
\hline 7 & 4085 & 3.1 & 246 & 1.1 & 153 & 1.0 & 1.5 & 1.5 \\
\hline 8 & 4561 & 2.1 & 254 & 0.9 & 138 & 1.2 & 1.0 & 1.5 \\
\hline Mean & 3925 & 3.1 & 264 & 0.9 & 136 & 1.2 & 1.5 & 1.6 \\
\hline$C V[\%]$ & 12.0 & 29.0 & 8.7 & 22.2 & 13.2 & 8.3 & 33.3 & 56.3 \\
\hline
\end{tabular}

Table 4: Backcalculation resutls with different load-time histories from Table 1.

(a)

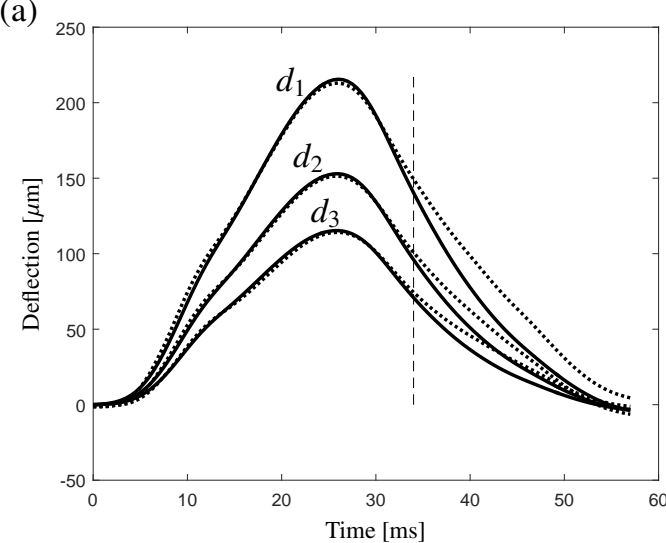

(b)

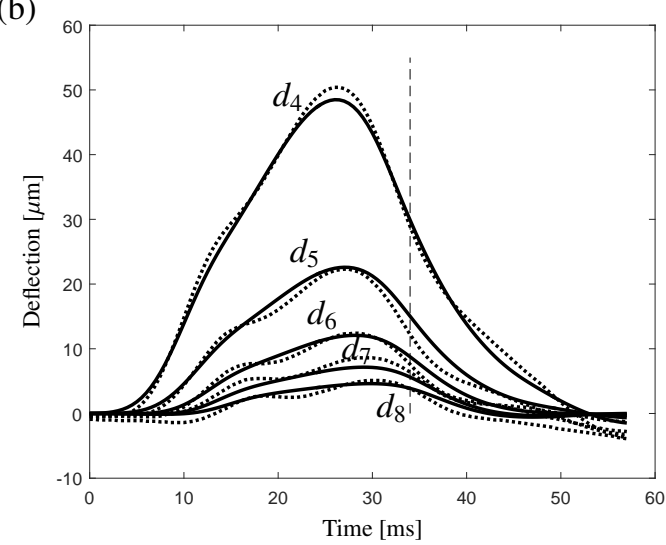

Figure 8: Measured (dotted) and modelled (solid) deflection histories for Test 6 (see Table 1).

(a)

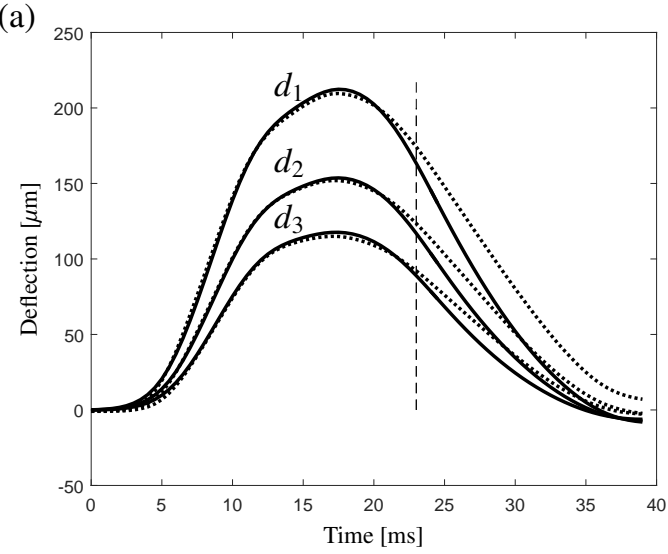

(b)

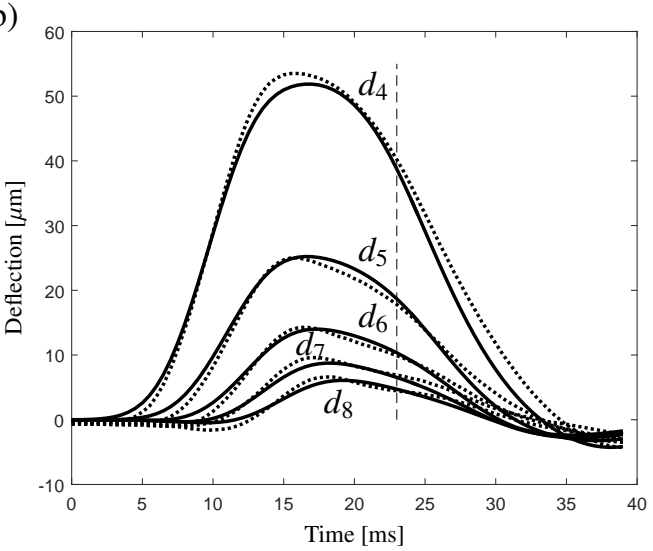

Figure 9: Measured (dotted) and modelled (solid) deflection histories for Test 8 (see Table 1). 
coefficient of variation for the damping parameters was nearly three times larger. From a statistical point of view the damping parameters are significantly different from zero with a t-statistic larger than 9 . As the actual properties of the tested pavement are only accessible via backcalculation, there are no reference values to compare against. Nevertheless, the backcalculated values are well within the bounds defined in Table 3 and are deemed reasonable and realistic from an engineering point of view.

As means of investigating the influence of different FWD load-time histories on backcalculated outcomes, three charts were prepared (see Figure 10) by combining data from Table 1 with data from Table 4. Figure 10(a) cross-plots moduli values and pulse duration across all peak loads, Figure 10(b) depicts moduli values versus peak loads across all pulse durations, and Figure 10(c) cross-plots damping values and pulse duration. As can be graphically noticed in the charts, some backcalculated parameters are influenced by the FWD load-time history. Specifically, the AC modulus $\left(E_{1}\right)$ displays slight increase with increasing peak load levels (Figure 10(b)); the positive trend is statistically significant within a confidence interval of $85 \%$. Similar sensitivity, but with opposite trend, is exhibited by the base and top of subgrade moduli $\left(E_{2}\right.$ and $\left.E_{3}^{0}\right)$; the negative trends are statistically significant within a confidence interval of $90 \%$ and $98 \%$, respectively. Furthermore, the subgrade modulus is increasing with increasing pulse duration (Figure 10(a)); the slope is positive with 95\% confidence level. As for layer damping, the $\mathrm{AC}$ is most sensitive, showing an increase in value with increase in pulse duration (slope is positive with 99.5\% confidence level). Subgrade damping is slightly increasing with increase in pulse duration (70\% confidence level) while base damping is uncorrelated with FWD pulse duration. High confidence levels for the trends indicate that the findings are not due to measurement repeatability issues which are of random nature.

The results of the investigation indicate that different FWD load-time histories produce different backcalculation parameters for the tested pavement. In other words, the optimized values of the model parameters had to be readjusted to best match the measured deflections depending on the pulse attributes. If backcalculation parameters differ when FWD pulse attributes are allowed to vary, then essentially new constitutive information is exposed under the different loading situations.

A possible enhancement of the model is a better representation of AC viscoelasticity. To further demonstrate this point consider the creep compliance function of the AC layer based on the Kelvin-Voigt representation

$$
D_{1}(t)=\frac{1}{E_{1}}\left(1-e^{-t / \eta_{1}}\right)
$$

In Figure 11(a) Equation (26) is plotted for all eight backcalculated results $E_{1}$ and $\eta_{1}$ from Table 4 (solid lines). Under the $\log -\log$ scale, the resulting curves appear concave. Also included in this Figure is the laboratory-measured creep compliance function (dotted line), which is sigmoidal in nature and plots as an S shaped curve. The two functions are dissimilar and only match in the average sense within time-frame considered.

The laboratory measured creep compliance curve was converted to the frequency domain according to the method described in Park and Schapery (1999). The derived norm of the complex modulus $\left|E_{1}^{*}\right|$ is shown in Figure 11(b) as a function of frequency (dotted line). This figure also includes the interconverted backcalculation results, i.e., the norm of the complex modulus for each

of the Kelvin-Voigt model: $\left|E_{1}^{*}\right|=E_{1} \sqrt{1+\omega^{2} \eta_{1}^{2}}$ (with values from Table 4). An FWD pseudo-frequency range is superposed onto the figure to illustrate the most relevant range for comparison (Ktari et al., (2014)).

\section{Conclusion}

The aim of this paper was to investigate the influence of different FWD load-time histories on backcalculation outcomes. An experiment was designed and carried out wherein an asphalt pavement was exposed to a variety of FWD tests within a short time. The tests differed from one another by the device settings in terms of drop height, dropped mass, and number of buffers. Initially, a new semi-analytic FWD loading model was shown to match well the diverse load-time histories generated in the experiment. Then, a new dynamic FE code was applied to backcalculate layer properties from the deflection histories. As a result of these two separate efforts, and despite the fact that one single pavement was experimentally investigated, some general conclusions can be made.

When an advanced dynamic FE pavement model is employed, combined with careful steps to ensure correct parameter convergence, backcalculated properties appear sensitive to the FWD pulse features. This finding suggests that modelling complexity is simplified. The rational here is that if a more intricate pavement model is employed then inferred properties based on any deflection test should remain identical when backcalculated. Possible enhancements in this connection include: better viscoelastic representation of the AC layer, allowance for nonlinear stress-dependent behaviour of the base and subgrade, and 
(a)

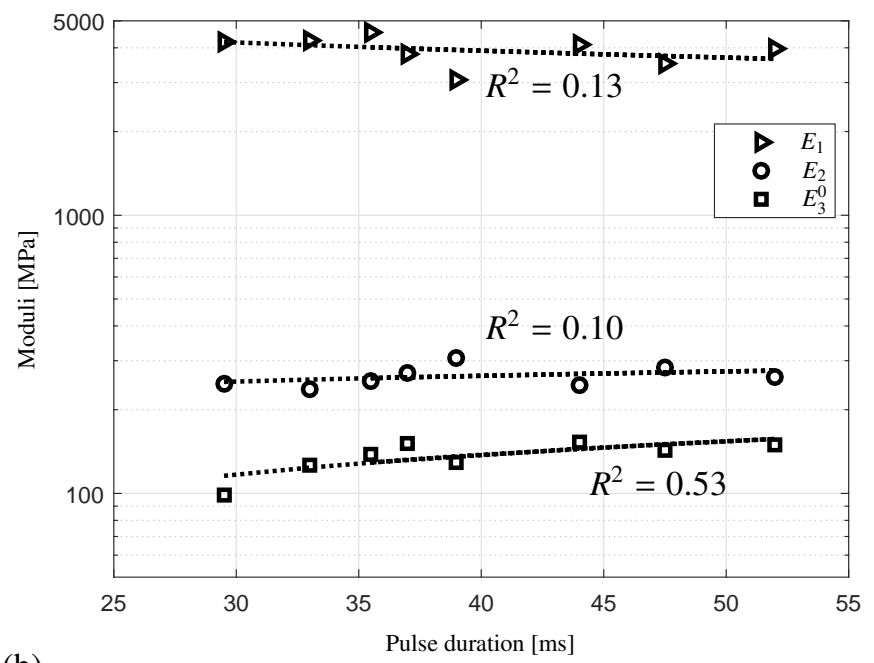

(b)

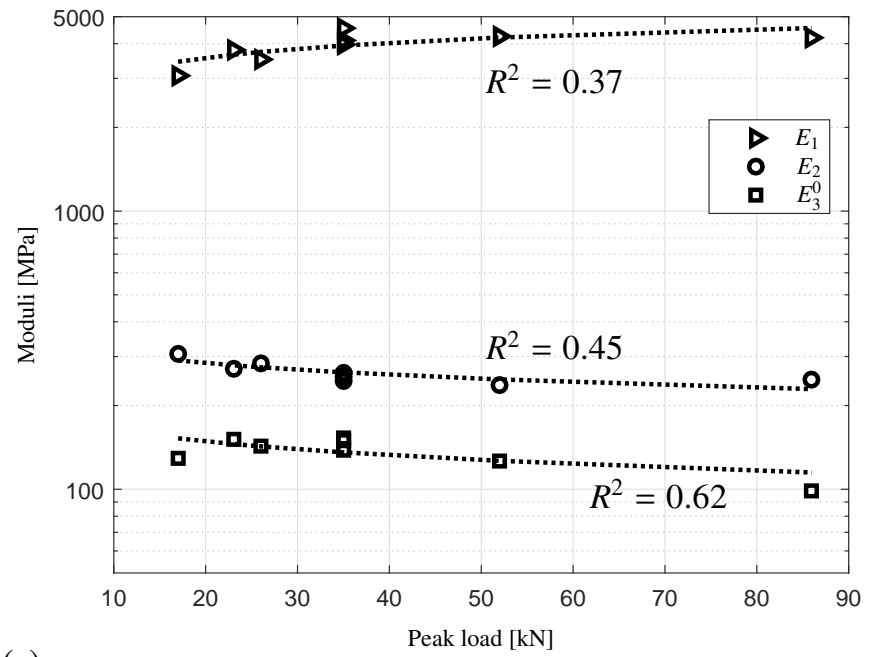

(c)

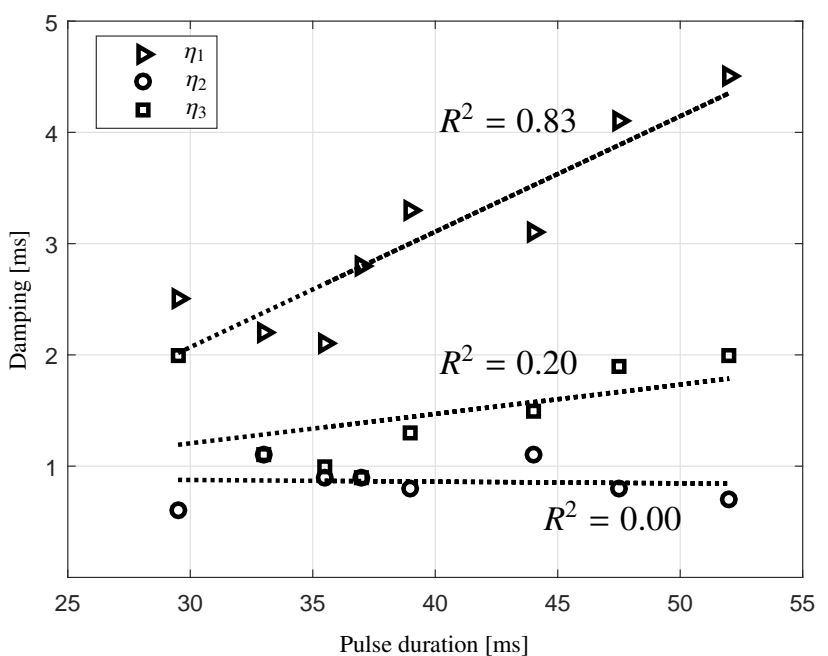

Figure 10: Backcalculation results: (a) moduli versus pulse duration, (b) moduli versus peak load, and (c) damping versus pulse duration. 

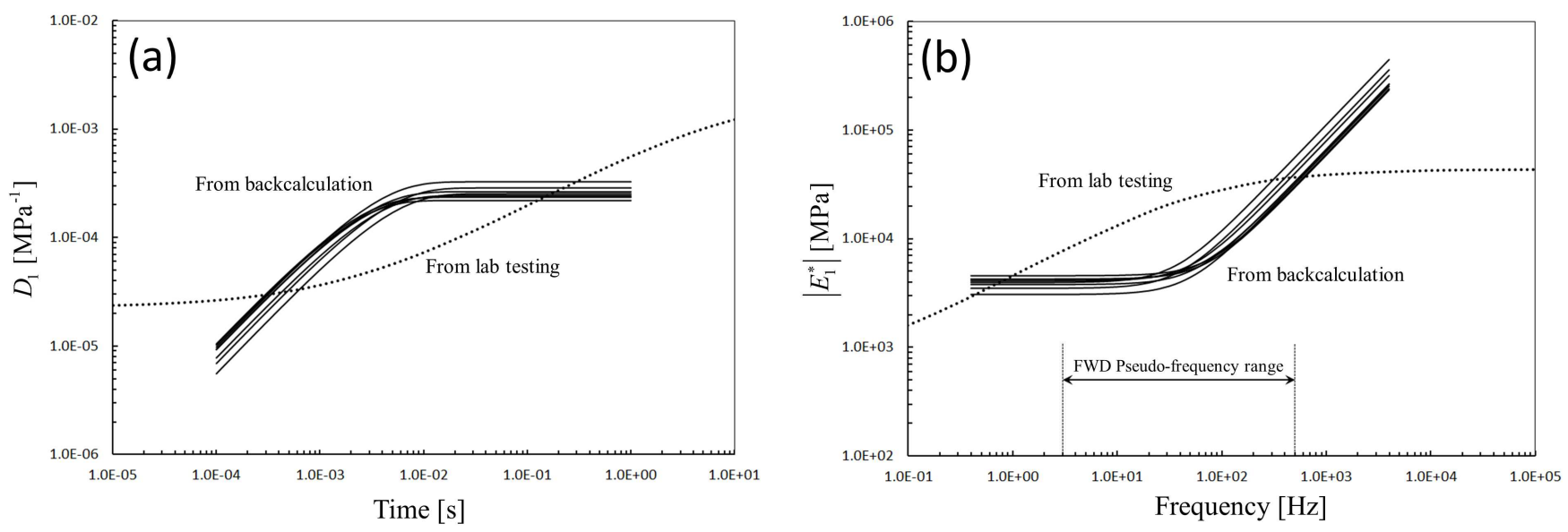

Figure 11: Backcalculated and measured AC layer properties; (a) creep compliance, and (b) norm of complex modulus.

incorporation of anisotropic response. The downside of complicating the forward modeling is that more unknown constitutive parameters will need to be evaluated.

However, the observed parameter sensitivity to FWD pulse attributes essentially means that new constitutive information is exposed under the different loading situations. Consequently, opportunity arises for increasing the modelling complexity as mentioned above because a wider calibration set becomes available to reliably infer the new/additional constitutive parameters. To achieve this, the testing should include diverse load-time histories, and the analysis should require simultaneous matching of all deflection histories. The new semi-analytic loading model that was offered herein for simulating FWD pulses could be utilized in this connection.

Consequently, based on the findings from this study, whenever advanced pavement layer characterization is sought, it is recommended that: (i) experimental attention be placed on generating diverse FWD pulse histories, and (ii) backcalculation be performed by the requirement to match all deflection histories, across all tested cases, simultaneously. Such an approach can potentially improve the ability for backcalculating AC mastercurves, where the analysis of a single FWD drop has been demonstrated to be insufficient (Varma et al. (2013); Kutay et al. (2011)). The expected improvements are due to basing the interpretation on a wider variety of pulse durations, and due to the diminished influence of random measurement errors in any single drop.

\section{Acknowledgements}

This work is supported by the Innovation Fund Denmark through the grant 'High-Speed Pavement Assessment Tool for Better and Safer Roads'. The FWD experiments were conducted in collaboration with Dynatest and laboratory testing was carried out with help from Matteo Pettinari at the Danish Road Directorate.

\section{References}

ASTM D4694-09 (2015). Standard test method for deflections with a falling-weight-type impulse load device. ASTM International, West Conshohocken, PA.

Augustesen, A., Liingaard, M., and Lade, P. V. (2004). Evaluation of time-dependent behavior of soils. International Journal of Geomechanics, 4(3):137-156.

Bolton, M. and Wilson, J. (1991). Soil stiffness and damping. Structural Dynamics, 1-2:209-216.

Bush, A. J. and Baladi, G. Y. (1989). Nondestructive testing of pavements and backcalculation of moduli. Technical Report STP1026, ASTM International, West Conshohocken, PA. 
Chatti, K., Ji, Y., and Harichandran, R. (2004). Dynamic time domain backcalculation of layer moduli, damping, and thicknesses in flexible pavements. Transportation Research Record: Journal of the Transportation Research Board, 1869:106116.

Cook, R., Malkus, D., and Plesha, M. (2002). Concepts and applications of finite element analysis. Wiley.

EN 12591:2009 (2009). Bitumen and bituminous binders. specifications for paving grade bitumens. BSI.

Ktari, R., Broutin, M., Picoux, B., Neji, J., and Petit, C. (2014). The benefits of using viscoelasticity in the dynamic simulation of heavy weight deflectometer tests. In Proceedings of the Twelfth International Conference on Computational Structures Technology, volume 106. Civil-Comp Press, Stirlingshire, Scotland.

Kutay, M., Chatti, K., and Lei, L. (2011). Backcalculation of dynamic modulus mastercurve from falling weight deflectometer surface deflections. Transportation Research Record: Journal of the Transportation Research Board, 2227:87-96.

Lee, H. S. (2014). Viscowave - a new solution for viscoelastic wave propagation of layered structures subjected to an impact load. International Journal of Pavement Engineering, 15(6):542-557.

Levenberg, E. (2014). Viscoelastic-viscoplastic characterization of unbound granular material. Advances in Civil Engineering Materials, 3(1):21-42.

Levenberg, E. and Michaeli, N. (2013). Viscoelastic characterisation of asphalt-aggregate mixes in diametral compression. Road Materials and Pavement Design, 14(S1):105-119.

Liingaard, M., Augustesen, A., and Lade, P. V. (2004). Characterization of models for time-dependent behavior of soils. International Journal of Geomechanics, 4(3):157-177.

Lukanen, E. O. (1992). Effects of buffers on falling weight deflectometer loadings and deflections (with discussion). Transportation Research Record, 1355:37-51.

Madsen, S. S. (2016). Dynamic Modeling of Pavements with Application to Deflection Measurements. PhD thesis, Technical University of Denmark, Kgs. Lyngby.

Mankovits, T., Huri, D., Kllai, I., Kocsis, I., and Szab, T. (2014). Material characterization and numerical simulation of a rubber bumper. International Journal of Mechanical, Aerospace, Industrial, Mechatronic and Manufacturing Engineering, 8(8):1367-1370.

Osyczka, A. (1978). Approach to multicriterion optimization problems for engineering design. Computer Methods in Applied Mechanics and Engineering, 15(3):309-333.

Park, S. W. and Schapery, R. A. (1999). Methods of interconversion between linear viscoelastic material functions. part I - a numerical method based prony series. International Journal of Solids and Structures, 36(11):1653-1675.

Quintus, H., Bush, A. I., and Baladi, G. (1994). Nondestructive testing of pavements and backcalculation of moduli: Second volume. Technical Report STP1198, ASTM International, West Conshohocken, PA.

Sebaaly, B., Davis, T. G., and Mamlouk, M. S. (1985). Dynamics of falling weight deflectometer. Journal of Transportation Engineering, 111(6):618-632.

Seed, H., Wong, R., Idriss, I., and Tokimatsu, K. (1986). Moduli and damping factors for dynamic analyses of cohesionless soils. Journal of Geotechnical Engineering, 112(11):1016-1032.

Shao, K.-Y., Roesset, J. M., and Stokoe, K. H. (1986). Dynamic Interpretation of Dynaflect and Falling Weight Deflectometer Tests on Pavement Systems. Center for Transportation Research, University of Texas at Austin.

Tayabji, S. and Lukanen, E. (2000). Nondestructive testing of pavements and backcalculation of moduli: Third volume. Technical Report STP1375, ASTM International, West Conshohocken, PA. 
Ullidtz, P. (1998). Modelling Flexible Pavement Response and Performance. Polyteknisk Forlag.

Ullidtz, P. and Stubstad, R. N. (1985). Analytical-empirical pavement evaluation using the falling weight deflectometer. Transportation Research Record: Journal of the Transportation Research Board, 1022:36-44.

Uzan, J. (1994). Dynamic linear backcalculation of pavement material parameters. Journal of Transportation Engineering, 120(1):109-126.

Uzan, J. and Lytton, R. L. (1990). Analysis of pressure distribution under falling weight deflectometer loading. Journal of Transportation Engineering, 116(2):246-250.

Varma, S., Kutay, M. E., and Chatti, K. (2013). Data requirements from falling weight deflectometer tests for accurate backcalculation of dynamic modulus master curve of asphalt pavements. Airfield and Highway Pavement 2013: Sustainable and Efficient Pavements - Proceedings of the 2013 Airfield and Highway Pavement Conference, pages 445-455.

Zou, D. G., Gong, T., Liu, J. M., and Kong, X. J. (2012). Shear modulus and damping ratio of gravel material. Applied Mechanics and Materials, 105-107:1426-1432. 